\title{
INTERPOLATING RATIONAL BÉZIER SPLINE CURVES WITH LOCAL SHAPE CONTROL
}

\author{
Alexander P. Pobegailo ${ }^{1}$ \\ ${ }^{1}$ Department of Applied Mathematics and Computer Science, Belarusian State \\ University, Minsk, Belarus
}

\begin{abstract}
The paper presents a technique for construction of $C^{n}$ interpolating rational Bézier spline curves by means of blending rational quadric Bézier curves. A class of polynomials which satisfy special boundary conditions is used for blending. Properties of the polynomials are considered. The constructed spline curves have local shape control that make them useful in such geometric applications as real-time trajectory generation and fast curve sketching.
\end{abstract}

\section{KEYWORDS}

Blending Curves, Rational Bézier curves, Interpolation, Splines, Interpolating Rational Splines

\section{INTRODUCTION}

Interpolating spline curves play important role in different geometric applications. This paper presents an approach to construction of interpolating rational Bézier spline curves with local control which have $C^{n}$ continuity. This property makes the spline curves suitable for using in different real-time geometric applications concerned with trajectory generation. A shape of the constructed spline curve can be modified by means of weights which are assigned to knot points of the spline curve. This feature enables using of the presented spline curves for fast sketching.

Segments of the presented spline curves are constructed by means of blending rational quadric Bézier curves represented in homogeneous coordinates. The blending is performed by means of special polynomials which are considered in the paper. The polynomials ensure a necessary parametric continuity of the designed spline curves. The presented approach can be considered as generalization of the approach to construction of interpolating spline curves in linear spaces considered by the author [1].

Firstly construction of spline curves by linear blending of parabolic arcs was proposed by Overhauser [2] and considered by Rogers and Adams [3]. Using linear blending of conics for construction of spline curves was considered by Chuan Sun [4]. Polynomial blending which ensures $G^{n}$ continuity is considered in other articles of Hartmann [5] and Meek, Walton [6]. Some other works concerned with interpolation with rational spline curves can be mentioned. Tai, Barsky and Loe presented an interpolation method that is based on blending a nonuniform rational B-spline curve with a singularly reparameterized linear spline [7]. Interpolating rational spline curves of cubic degree with shape control are considered in works [8-10]. Rational cubic spline curves with $G^{2}$ continuity is considered in the work [11]. Weighted rational cubic spline interpolation and its application are considered in the articles [12-15].

DOI : 10.5121/ijcga.2013.3401 


\section{BLending Polynomials}

The purpose of this section is to define polynomials which will be used for blending of parametric curves. For this purpose consider the following knot sequences

$$
(\underbrace{0,0, \ldots, 0,1,1, \ldots, 1}_{n})
$$

and define the following polynomials:

$$
w_{n}(u)=\sum_{i=n}^{2 n-1} b_{2 n-1, i}(u), u \in[0,1],
$$

where $b_{n, m}(u)$ are Bernstein polynomials

$$
b_{n, m}(u)=\frac{n !}{m !(n-m) !}(1-u)^{n-m} u^{m}
$$

It follows from this definition that the polynomials $w_{n}(u)$ meet the following boundary conditions:

$$
\begin{aligned}
& w_{n}(0)=0, w_{n}(1)=1, \\
& w_{n}{ }^{(m)}(0)=w_{n}{ }^{(m)}(1)=0, \forall m \in\{1,2, \ldots, n-1\} .
\end{aligned}
$$

The polynomials $w_{n}(u)$ have the following properties:

$$
\begin{aligned}
& w_{n}(u)+w_{n}(1-u)=1, \\
& w_{n}(1 / 2+v)+w_{n}(1 / 2-v)=1
\end{aligned}
$$

which follow from the property

$$
\sum_{m=0}^{n} b_{n, m}(u)=1
$$

of Bernstein polynomials. It follows from Equation (2.4) that the polynomials $w_{n}(u)$ are symmetric with respect to the point $(1 / 2,1 / 2)$.

Besides it can be proven that

$$
\lim _{n \rightarrow \infty} \int_{0}^{1 / 2} w_{n}(u) d u=0
$$

and that the polynomial $w_{n}(u)$ is a minimum of the functional

$$
J_{n}(f)=\int_{0}^{1}\left|f^{(n)}(u)\right|^{2} d u, n \in N .
$$

Proofs of the properties can be found in the paper [1]. Figure 1 shows graphs of the polynomials $w_{n}(u)$.

The following polynomials of lower degrees:

$$
\begin{aligned}
& w_{1}(u)=u, \\
& w_{2}(u)=3(1-u) u^{2}+u^{3}, \\
& w_{3}(u)=10(1-u)^{2} u^{3}+5(1-u) u^{4}+u^{5}
\end{aligned}
$$


are often used in geometric applications.

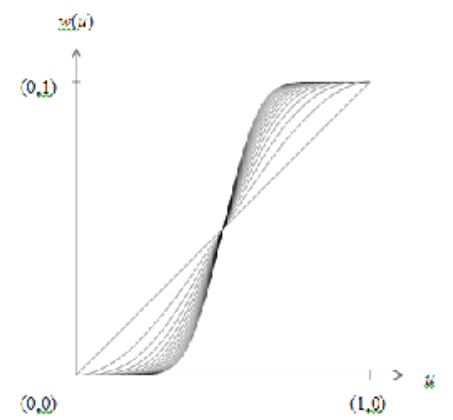

Figure 1. Graphs of the polynomials $w_{n}(u)$

The polynomials $w_{n}(u)$ were introduced by the author $[16,17]$. The representation of these polynomials $w_{n}(u)$ by means of Bernstein polynomials was proposed by Wiltsche [18].

\section{BLEnding Conic ArCS}

Consider two conic arcs $\boldsymbol{p}_{i}(u), i \in\{1,2\}$, which are represented by means of rational Bézier curves and have common boundary points that is

$$
\boldsymbol{p}_{i}(u)=\frac{(1-u)^{2} w_{0} \boldsymbol{p}_{0}+2(1-u) u w_{i, 1} \boldsymbol{p}_{i, 1}+u^{2} w_{2} \boldsymbol{p}_{2}}{(1-u)^{2} w_{0}+2(1-u) u w_{i, 1}+u^{2} w_{2}}, u \in[0,1]
$$

The problem is to construct a parametric curve $\boldsymbol{p}(u)$ which has the following boundary points:

$$
\boldsymbol{p}(0)=\boldsymbol{p}_{1}(0)=\boldsymbol{p}_{0}, \boldsymbol{p}(1)=\boldsymbol{p}_{2}(1)=\boldsymbol{p}_{2}
$$

and satisfies the following boundary conditions:

$$
\boldsymbol{p}^{(m)}(0)=\boldsymbol{p}_{1}{ }^{(m)}(0), \boldsymbol{p}^{(m)}(1)=\boldsymbol{p}_{2}^{(m)}(1), \forall m \in\{1,2, \ldots, n\},
$$

where $n \in N$. The parametric curve $\boldsymbol{p}(u)$ which satisfies Equations (3.2) and (3.3) is called a parametric curve blending the parametric curves $\boldsymbol{p}_{1}(u)$ and $\boldsymbol{p}_{2}(u)$.

In order to solve the problem represent the parametric curves $\boldsymbol{p}_{i}(u), i \in\{1,2\}$, using homogeneous coordinates as follows:

$$
\boldsymbol{x}_{i}(u)=(1-u)^{2} \boldsymbol{x}_{0}+2(1-u) u \boldsymbol{x}_{i, 1}+u^{2} \boldsymbol{x}_{2}, u \in[0,1],
$$

where the points $\boldsymbol{x}_{0}, \boldsymbol{x}_{i, 1}$ and $\boldsymbol{x}_{2}$ have the corresponding weight coordinates $w_{0}, w_{i, 1}$ and $w_{2}$. Then define the parametric curve $\boldsymbol{x}(u)$ as follows:

$$
\boldsymbol{x}(u)=\left(1-w_{n}(u)\right) \boldsymbol{x}_{1}(u)+w_{n}(u) \boldsymbol{x}_{2}(u), u \in[0,1],
$$

It follows from this definition that the corresponding parametric curve $\boldsymbol{p}(u)$ which is obtained from the parametric curve $\boldsymbol{x}(u)$ by transition to Cartesian coordinates has the following rational representation:

$$
\boldsymbol{p}(u)=\frac{\left(1-w_{n}(u)\right) \boldsymbol{r}_{1}(u)+w_{n}(u) \boldsymbol{r}_{2}(u)}{\left(1-w_{n}(u)\right) r_{1}(u)+w_{n}(u) r_{2}(u)}, u \in[0,1],
$$

where 
International Journal of Computer Graphics \& Animation (IJCGA) Vol.3, No.4, October 2013

$$
\begin{aligned}
& \boldsymbol{r}_{i}(u)=(1-u)^{2} w_{0} \boldsymbol{p}_{0}+2(1-u) u w_{i, 1} \boldsymbol{p}_{i, 1}+u^{2} w_{2} \boldsymbol{p}_{2}, i \in\{1,2\}, \\
& r_{i}(u)=(1-u)^{2} w_{0}+2(1-u) u w_{i, 1}+u^{2} w_{2}, i \in\{1,2\} .
\end{aligned}
$$

It follows form the definition of the polynomials $w_{n}(u)$ that the parametric curve $\boldsymbol{p}(u)$ satisfies conditions (3.2) because

and

$$
\boldsymbol{p}(0)=\frac{\left(1-w_{n}(0)\right) \boldsymbol{r}_{1}(0)+w_{n}(0) \boldsymbol{r}_{2}(0)}{\left(1-w_{n}(0)\right) r_{1}(0)+w_{n}(0) r_{2}(0)}=\frac{\boldsymbol{r}_{1}(0)}{r_{1}(0)}=\boldsymbol{p}_{1}(0)=\boldsymbol{p}_{0}
$$

$$
\boldsymbol{p}(1)=\frac{\left(1-w_{n}(1)\right) \boldsymbol{r}_{1}(1)+w_{n}(1) \boldsymbol{r}_{2}(1)}{\left(1-w_{n}(1)\right) r_{1}(0)+w_{n}(1) r_{2}(1)}=\frac{\boldsymbol{r}_{2}(1)}{r_{2}(1)}=\boldsymbol{p}_{2}(1)=\boldsymbol{p}_{2} .
$$

Derivatives of the parametric curve $\boldsymbol{p}(u)$ depend on derivatives of the numerator and denominator of Equation (3.5). In order to simplify further considerations introduce the following denotations:

$$
\begin{aligned}
& \boldsymbol{r}(u)=\left(1-w_{n}(u)\right) \boldsymbol{r}_{1}(u)+w_{n}(u) \boldsymbol{r}_{2}(u), u \in[0,1], \\
& r(u)=\left(1-w_{n}(u)\right) r_{1}(u)+w_{n}(u) r_{2}(u), u \in[0,1] .
\end{aligned}
$$

Now determine derivatives of the numerator $\boldsymbol{r}(u)$ and denominator $r(u)$. It is obtained using Leibnitz's formula that

$$
\begin{aligned}
& \boldsymbol{r}^{(m)}(u)=\sum_{i=0}^{m} \frac{m !}{i !(m-i) !}\left(\left(1-w_{n}(u)\right)^{(i)} \boldsymbol{r}_{1}^{(m-i)}(u)+\left(w_{n}(u)\right)^{(i)} \boldsymbol{r}_{2}^{(m-i)}(u),\right. \\
& r^{(m)}(u)=\sum_{i=0}^{m} \frac{m !}{i !(m-i) !}\left(\left(1-w_{n}(u)\right)^{(i)} r_{1}^{(m-i)}(u)+\left(w_{n}(u)\right)^{(i)} r_{2}^{(m-i)}(u)\right.
\end{aligned}
$$

for any $m \in N$. Substitution of Equations (2.2) into these equations yields that the derivatives have the following values at the boundaries of the interval $\left[0,{ }^{\circ} 1\right]$ :

$$
\begin{aligned}
& \boldsymbol{r}^{(m)}(0)=\left(1-w_{n}(0)\right) \boldsymbol{r}_{1}^{(m)}(0)+w_{n}(0) \boldsymbol{r}_{2}^{(m)}(0)=\boldsymbol{r}_{1}^{(m)}(0), \\
& \boldsymbol{r}^{(m)}(1)=\left(1-w_{n}(1)\right) \boldsymbol{r}_{1}^{(m)}(0)+w_{n}(1) \boldsymbol{r}_{2}^{(m)}(1)=\boldsymbol{r}_{2}^{(m)}(1)
\end{aligned}
$$

and analogously

$$
\begin{aligned}
& r^{(m)}(0)=\left(1-w_{n}(0)\right) r_{1}^{(m)}(0)+w_{n}(0) r_{2}^{(m)}(0)=r_{1}^{(m)}(0), \\
& r^{(m)}(1)=\left(1-w_{n}(1)\right) r_{1}^{(m)}(0)+w_{n}(1) r_{2}^{(m)}(1)=r_{2}^{(m)}(1)
\end{aligned}
$$

for any $\forall m \in\{1,2, \ldots, n-1\}$. Now show the derivatives of the order $n$ also have necessary values at the boundaries of the domain $\left[0,{ }^{\circ} 1\right]$. It can be seen that

$$
\begin{aligned}
& \boldsymbol{r}^{(n)}(0)=-w_{n}^{(n)}(0) \boldsymbol{r}_{1}(0)+\left(1-w_{n}(0)\right) \boldsymbol{r}_{1}^{(n)}(0)+w_{n}^{(n)}(0) \boldsymbol{r}_{2}(0)+w_{n}(0) \boldsymbol{r}_{2}^{(n)}(0)=\boldsymbol{r}_{1}^{(n)}(0) \\
& \boldsymbol{r}^{(n)}(1)=-w_{n}^{(n)}(1) \boldsymbol{r}_{1}(1)+\left(1-w_{n}(1)\right) \boldsymbol{r}_{1}^{(n)}(1)+w_{n}^{(n)}(1) \boldsymbol{r}_{2}(1)+w_{n}(1) \boldsymbol{r}_{2}^{(n)}(1)=\boldsymbol{r}_{2}^{(n)}(1)
\end{aligned}
$$

and analogously 


$$
r^{(n)}(0)=r_{1}^{(n)}(0), r^{(n)}(1)=r_{2}^{(n)}(1)
$$

Thus it is proved that

$$
\begin{aligned}
& \boldsymbol{r}^{(m)}(0)=\boldsymbol{r}_{1}^{(m)}(0), \boldsymbol{r}^{(m)}(1)=\boldsymbol{r}_{2}^{(m)}(1), \\
& r^{(m)}(0)=r_{1}^{(m)}(0), r^{(m)}(1)=r_{2}^{(m)}(1), \forall m \in\{1,2, \ldots, n\} .
\end{aligned}
$$

Using obtained results compute higher order derivatives of the parametric curve $\boldsymbol{p}(u)$. It follows form Equation (3.5) using introduced denotations for the numerator and denominator that

$$
r(u) \boldsymbol{p}(u)=\boldsymbol{r}(u), u \in[0,1] .
$$

Differentiation of the last equation using Leibnitz's formula yields that

$$
\sum_{i=0}^{m} \frac{m !}{i !(m-i) !} r^{(i)}(u) \boldsymbol{p}^{(m-i)}(u)=\boldsymbol{r}^{(m)}(u), m \in N .
$$

Substitution of Equations (3.6), (3.8) and (3.7), (3.9) into the last equation yields the following values of derivatives at the boundaries of the interval $\left[0,{ }^{\circ} 1\right]$ :

$$
\begin{aligned}
& \sum_{i=0}^{m} \frac{m !}{i !(m-i) !} r_{1}^{(i)}(0) \boldsymbol{p}^{(m-i)}(0)=\boldsymbol{r}_{1}^{(m)}(0), \\
& \sum_{i=0}^{m} \frac{m !}{i !(m-i) !} r_{2}^{(i)}(1) \boldsymbol{p}^{(m-i)}(1)=\boldsymbol{r}_{2}^{(m)}(1) .
\end{aligned}
$$

On the other hand it follows from Equations (3.1) that

$$
r_{i}(u) \boldsymbol{p}_{i}(u)=\boldsymbol{r}_{i}(u), i \in\{1,2\},
$$

and therefore derivatives of the parametric curves $\boldsymbol{p}_{i}(u), i \in\{1,2\}$, satisfy the following equations:

$$
\begin{aligned}
& \sum_{i=0}^{m} \frac{m !}{i !(m-i) !} r_{1}^{(i)}(0) \boldsymbol{p}_{1}{ }^{(m-i)}(0)=\boldsymbol{r}_{1}^{(m)}(0) \\
& \sum_{i=0}^{m} \frac{m !}{i !(m-i) !} r_{2}^{(i)}(1) \boldsymbol{p}_{2}{ }^{(m-i)}(1)=\boldsymbol{r}_{2}^{(m)}(1)
\end{aligned}
$$

Show that Equations (3.10) and (3.12) are equivalent. Consider the first derivatives of the parametric curves $\boldsymbol{p}(u)$ and $\boldsymbol{p}_{1}(u)$. It follows from Equations (3.10) and (3.12) that the first derivatives satisfy the following two equations:

$$
r_{1}(0) \boldsymbol{p}^{(1)}(0)+r_{1}^{(1)}(0) \boldsymbol{p}(0)=\boldsymbol{r}_{1}^{(1)}(0), r_{1}(0) \boldsymbol{p}_{1}{ }^{(1)}(0)+r_{1}^{(1)}(0) \boldsymbol{p}_{1}(0)=\boldsymbol{r}_{1}{ }^{(1)}(0) .
$$

It follows from these two equations taking into account Equations (3.2) that

$$
\boldsymbol{p}^{(1)}(0)=\boldsymbol{p}_{1}^{(1)}(0) \text {. }
$$

Now assume that the following equation: 


$$
\boldsymbol{p}^{(m-1)}(0)=\boldsymbol{p}_{1}^{(m-1)}(0)
$$

is also fulfilled. Then consider the $m$-th order derivatives of the parametric curves $\boldsymbol{p}(u)$ and $p_{1}(u)$. It follows from Equations (3.10) and (3.12) that the $m$-th order derivatives satisfy the following equation:

$$
\sum_{i=0}^{m} \frac{m !}{i !(m-i) !} r_{1}^{(i)}(0) \boldsymbol{p}^{(m-i)}(0)=\sum_{i=0}^{m} \frac{m !}{i !(m-i) !} r_{1}^{(i)}(0) \boldsymbol{p}_{1}^{(m-i)}(0)
$$

which is equivalent to the equation

$$
\begin{aligned}
& r_{1}(0) \boldsymbol{p}^{(m)}(0)+\sum_{i=0}^{m-1} \frac{(m-1) !}{i !(m-1-i) !} r_{1}^{(i)}(0) \boldsymbol{p}^{(m-1-i)}(0)= \\
& =r_{1}(0) \boldsymbol{p}_{1}{ }^{(m)}(0)+\sum_{i=0}^{m-1} \frac{(m-1) !}{i !(m-1-i) !} r_{1}^{(i)}(0) \boldsymbol{p}_{1}{ }^{(m-1-i)}(0) .
\end{aligned}
$$

It follows from the last equation taking into account the assumption that

$$
\boldsymbol{p}^{(m)}(0)=\boldsymbol{p}_{1}^{(m)}(0)
$$

Therefore the last equation is fulfilled for all $m \in N$ by the principle of mathematical induction. Analogously it can be proven using Equations (3.11) and (3.13) that

$$
\boldsymbol{p}^{(m)}(1)=\boldsymbol{p}_{2}^{(m)}(1)
$$

for all $m \in N$. Thus Equations (3.3) are also fulfilled.

Figure 2 shows some curves constructed by means of blending two conic arcs with the polynomials $w_{n}(u)$.

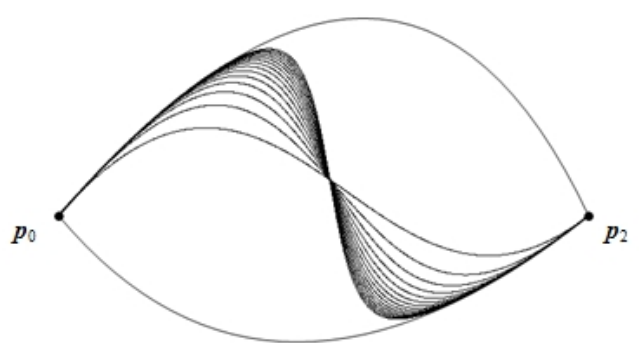

Figure 2. Blending conic arcs by means of the polynomials $w_{n}(u)$

\section{BÉZIER REPRESENTATION OF BLENDEd CONIC ARCS}

The purpose of this section is to obtain a rational Bézier representation of the blending parametric curve $\boldsymbol{p}(u)$ described by Equation (3.5). In order to solve the problem consider a homogeneous representation $\boldsymbol{x}(u)$ of the parametric curve $\boldsymbol{p}(u)$ which is described by Equation (3.6). Using Equation (2.3) the homogeneous representation can be transformed as follows:

$$
\begin{aligned}
& \boldsymbol{x}(u)=\left(1-w_{n}(u)\right) \boldsymbol{x}_{1}(u)+w_{n}(u) \boldsymbol{x}_{2}(u)= \\
& =w_{n}(1-u) \boldsymbol{x}_{1}(u)+w_{n}(u) \boldsymbol{x}_{2}(u)=
\end{aligned}
$$


International Journal of Computer Graphics \& Animation (IJCGA) Vol.3, No.4, October 2013

$$
\begin{aligned}
& =\sum_{k=0}^{n-1} b_{2 n-1, k}(u) \boldsymbol{x}_{1}(u)+\sum_{k=n}^{2 n-1} b_{2 n-1, k}(u) \boldsymbol{x}_{2}(u)= \\
& =\sum_{k=0}^{n-1} b_{2 n-1, k}(u)\left(b_{2,0}(u) \boldsymbol{x}_{0}+b_{2,1}(u) \boldsymbol{x}_{1,1}+b_{2,2}(u) \boldsymbol{x}_{2}\right)+ \\
& +\sum_{k=n}^{2 n-1} b_{2 n-1, k}(u)\left(b_{2,0}(u) \boldsymbol{x}_{0}+b_{2,1}(u) \boldsymbol{x}_{2,1}+b_{2,2}(u) \boldsymbol{x}_{2}\right)= \\
& =\sum_{k=0}^{2 n-1} b_{2 n-1, k}(u) b_{2,0}(u) \boldsymbol{x}_{0}+\sum_{k=0}^{n-1} b_{2 n-1, k}(u) b_{2,1}(u) \boldsymbol{x}_{1,1}+ \\
& +\sum_{k=n}^{2 n-1} b_{2 n-1, k}(u) b_{2,1}(u) \boldsymbol{x}_{2,1}+\sum_{k=0}^{2 n-1} b_{2 n-1, k}(u) b_{2,2}(u) \boldsymbol{x}_{2}= \\
& =\sum_{k=0}^{2 n-1} b_{2 n+1, k}(u) c_{0, k} \boldsymbol{x}_{0}+\sum_{k=1}^{n} b_{2 n+1, k}(u) c_{1, k} \boldsymbol{x}_{1,1}+ \\
& +\sum_{k=n+1}^{2 n} b_{2 n+1, k}(u) c_{1, k} \boldsymbol{x}_{2,1}+\sum_{k=2}^{2 n+1} b_{2 n+1, k}(u) c_{2, k} \boldsymbol{x}_{2}
\end{aligned}
$$

Where

$$
\begin{aligned}
& c_{0, k}=\frac{(2 n-k)(2 n-k+1)}{2 n(2 n+1)}, 0 \leq k \leq 2 n-1, \\
& c_{1, k}=\frac{k(2 n-k+1)}{n(2 n+1)}, 1 \leq k \leq 2 n, \\
& c_{2, k}=\frac{(k-1) k}{2 n(2 n+1)}, 2 \leq k \leq 2 n+1 .
\end{aligned}
$$

It follows from the last equations that the parametric curve $\boldsymbol{x}(u)$ has the following Bézier representation:

$$
\begin{aligned}
& \boldsymbol{x}(u)=b_{2 n+1,0}(u) \boldsymbol{x}_{0}+b_{2 n+1,1}(u)\left(c_{0,1} \boldsymbol{x}_{0}+c_{1,1} \boldsymbol{x}_{1,1}\right)+ \\
& +\sum_{k=2}^{n} b_{2 n+1, k}(u)\left(c_{0, k} \boldsymbol{x}_{0}+c_{1, k} \boldsymbol{x}_{1,1}+c_{2, k} \boldsymbol{x}_{2}\right)+ \\
& +\sum_{k=n+1}^{2 n-1} b_{2 n+1, k}(u)\left(c_{0, k} \boldsymbol{x}_{0}+c_{1, k} \boldsymbol{x}_{2,1}+c_{2, k} \boldsymbol{x}_{2}\right)+ \\
& +b_{2 n+1,2 n}(u)\left(c_{1,2 n} \boldsymbol{x}_{2,1}+c_{2,2 n} \boldsymbol{x}_{2}\right)+b_{2 n+1,2 n+1}(u) \boldsymbol{x}_{2} .
\end{aligned}
$$

Then transition to Cartesian coordinates yields that the blending parametric curve $\boldsymbol{p}(u)$ has the following rational Bézier representation:

$$
\boldsymbol{p}(u)=\frac{\boldsymbol{r}(u)}{r(u)}, u \in[0,1],
$$

where

$$
\begin{aligned}
& \boldsymbol{r}(u)=b_{2 n+1,0}(u) w_{0} \boldsymbol{p}_{0}+b_{2 n+1,1}(u)\left(c_{0,1} w_{0} \boldsymbol{p}_{0}+c_{1,1} w_{1,1} \boldsymbol{p}_{1,1}\right)+ \\
& +\sum_{k=2}^{n} b_{2 n+1, k}(u)\left(c_{0, k} w_{0} \boldsymbol{p}_{0}+c_{1, k} w_{1,1} \boldsymbol{p}_{1,1}+c_{2, k} w_{2} \boldsymbol{p}_{2}\right)+ \\
& +\sum_{k=n+1}^{2 n-1} b_{2 n+1, k}(u)\left(c_{0, k} w_{0} \boldsymbol{p}_{0}+c_{1, k} w_{2,1} \boldsymbol{p}_{2,1}+c_{2, k} w_{2,2} \boldsymbol{p}_{2}\right)+
\end{aligned}
$$


International Journal of Computer Graphics \& Animation (IJCGA) Vol.3, No.4, October 2013

$$
+b_{2 n+1,2 n}(u)\left(c_{1,2 n} w_{2,1} \boldsymbol{p}_{2,1}+c_{2,2 n} w_{2} \boldsymbol{p}_{2}\right)+b_{2 n+1,2 n+1}(u) w_{2} \boldsymbol{p}_{2}
$$

and

$$
\begin{aligned}
& r(u)=b_{2 n+1,0}(u) w_{0}+b_{2 n+1,1}(u)\left(c_{0,1} w_{0}+c_{1,1} w_{1,1}\right)+ \\
& +\sum_{k=2}^{n} b_{2 n+1, k}(u)\left(c_{0, k} w_{0}+c_{1, k} w_{1,1}+c_{2, k} w_{2}\right)+ \\
& +\sum_{k=n+1}^{2 n-1} b_{2 n+1, k}(u)\left(c_{0, k} w_{0}+c_{1, k} w_{2,1}+c_{2, k} w_{2,2}\right)+ \\
& +b_{2 n+1,2 n}(u)\left(c_{1,2 n} w_{2,1}+c_{2,2 n} w_{2}\right)+b_{2 n+1,2 n+1}(u) w_{2}
\end{aligned}
$$

For example, the numerator and denominator of cubic and quintic blending parametric curves have the following Bézier representations:

$$
\begin{aligned}
& \boldsymbol{r}(u)=b_{3,0}(u) w_{0} \boldsymbol{p}_{0}+b_{3,1}(u) \frac{1}{3}\left(w_{0} \boldsymbol{p}_{0}+2 w_{1,1} \boldsymbol{p}_{1,1}\right)+ \\
& +b_{3,2}(u) \frac{1}{3}\left(2 w_{2,1} \boldsymbol{p}_{2,1}+w_{2} \boldsymbol{p}_{2}\right)+b_{3,3}(u) w_{2} \boldsymbol{p}_{2} \\
& r(u)=b_{3,0}(u) w_{0}+b_{3,1}(u) \frac{1}{3}\left(w_{0}+2 w_{1,1}\right)+b_{3,2}(u) \frac{1}{3}\left(2 w_{2,1}+w_{2}\right)+b_{3,3}(u) w_{2}
\end{aligned}
$$

and

$$
\begin{aligned}
& \boldsymbol{r}(u)=b_{5,0}(u) w_{0} \boldsymbol{p}_{0}+b_{5,1}(u) \frac{1}{5}\left(3 w_{0} \boldsymbol{p}_{0}+2 w_{1,1} \boldsymbol{p}_{1,1}\right)+ \\
& +b_{5,2}(u) \frac{1}{10}\left(3 w_{0} \boldsymbol{p}_{0}+6 w_{1,1} \boldsymbol{p}_{1,1}+w_{2} \boldsymbol{p}_{2}\right)+b_{5,3}(u) \frac{1}{10}\left(w_{0} \boldsymbol{p}_{0}+6 w_{2,1} \boldsymbol{p}_{2,1}+3 w_{2} \boldsymbol{p}_{2}\right)+ \\
& +b_{5,4}(u) \frac{1}{5}\left(2 w_{2,1} \boldsymbol{p}_{2,1}+3 w_{2} \boldsymbol{p}_{2}\right)+b_{5,5}(u) w_{2} \boldsymbol{p}_{2} \\
& r(u)=b_{5,0}(u) w_{0}+b_{5,1}(u) \frac{1}{5}\left(3 w_{0}+2 w_{1,1}\right)+ \\
& +b_{5,2}(u) \frac{1}{10}\left(3 w_{0}+6 w_{1,1}+w_{2}\right)+b_{5,3}(u) \frac{1}{10}\left(w_{0}+6 w_{2,1}+3 w_{2}\right)+ \\
& +b_{5,4}(u) \frac{1}{5}\left(2 w_{2,1}+3 w_{2}\right)+b_{5,5}(u) w_{2}
\end{aligned}
$$

respectively.

\section{Construction of Two Smoothly Joined Conic ArCs}

The purpose of this section is to introduce analytical expressions for construction smoothly joined conic arcs. The expressions will be used for construction spline curves in the next section. Consider three distinct points $\boldsymbol{p}_{0}, \boldsymbol{p}_{1}$, and $\boldsymbol{p}_{2}$ with the corresponding weights $w_{0}, w_{1}$ and $w_{2}$. The problem is to construct two conic $\operatorname{arcs} \boldsymbol{p}_{i}(u), i \in\{1,2\}$, which are represented by means of rational Bézier curves

$$
\begin{aligned}
& \boldsymbol{p}_{1}(u)=\frac{(1-u)^{2} w_{0} \boldsymbol{p}_{0}+2(1-u) u w_{1,1} \boldsymbol{p}_{1,1}+u^{2} w_{1} \boldsymbol{p}_{1}}{(1-u)^{2} w_{0}+2(1-u) u w_{1,1}+u^{2} w_{1}}, u \in[0,1], \\
& \boldsymbol{p}_{2}(u)=\frac{(1-u)^{2} w_{1} \boldsymbol{p}_{1}+2(1-u) u w_{2,1} \boldsymbol{p}_{2,1}+u^{2} w_{2} \boldsymbol{p}_{2}}{(1-u)^{2} w_{0}+2(1-u) u w_{2,1}+u^{2} w_{2}}, u \in[0,1]
\end{aligned}
$$

and are smoothly joined at the common point $\boldsymbol{p}_{1}$ that is 


$$
\boldsymbol{p}_{1}^{(n)}(1)=\boldsymbol{p}_{2}{ }^{(n)}(0), n \in N
$$

In order to find control points $\boldsymbol{p}_{1,1}$, and $\boldsymbol{p}_{2,1}$ which ensure the necessary smooth junction represent the conic $\operatorname{arcs} \operatorname{arcs} \boldsymbol{p}_{1}(u)$ and $\boldsymbol{p}_{2}(u)$ using homogeneous coordinates as follows

$$
\begin{aligned}
& \boldsymbol{x}_{1}(u)=(1-u)^{2} \boldsymbol{x}_{0}+2(1-u) u \boldsymbol{x}_{1,1}+u^{2} \boldsymbol{x}_{1}, u \in[0,1], \\
& \boldsymbol{x}_{2}(u)=(1-u)^{2} \boldsymbol{x}_{1}+2(1-u) u \boldsymbol{x}_{2,1}+u^{2} \boldsymbol{x}_{2}, u \in[0,1] .
\end{aligned}
$$

The conic $\operatorname{arcs} \operatorname{arcs} \boldsymbol{x}_{1}(u)$ and $\boldsymbol{x}_{2}(u)$ are smoothly joined at the point $\boldsymbol{x}_{1}$ only provided that the following two conditions:

$$
x_{1}^{\prime}(1)=x_{2}^{\prime}(0), x_{1}^{\prime \prime}(1)=x_{2}^{\prime \prime}(0)
$$

are fulfilled. Resolution of these equations yields the following values of unknown control points $\boldsymbol{x}_{1,1}$ and $\boldsymbol{x}_{2,2}$ of the quadric Bézier curves $\boldsymbol{x}_{1}(u)$ and $\boldsymbol{x}_{2}(u)$ :

$$
x_{1,1}=x_{1}-\frac{x_{2}-x_{0}}{4}, x_{2,1}=x_{1}+\frac{x_{2}-x_{0}}{4} .
$$

The values of knot points $\boldsymbol{p}_{1,1}$, and $\boldsymbol{p}_{2,1}$ can be obtained from these equations by transition to transition to Cartesian coordinates as follows:

$$
\begin{aligned}
& \boldsymbol{p}_{1,1}=w_{1} \boldsymbol{p}_{1}-\frac{w_{2} \boldsymbol{p}_{2}-w_{0} \boldsymbol{p}_{0}}{4}, \boldsymbol{p}_{2,1}=w_{1} \boldsymbol{p}_{1}+\frac{w_{2} \boldsymbol{p}_{2}-w_{0} \boldsymbol{p}_{0}}{4} . \\
& w_{1,1}=w_{1}-\frac{w_{2}-w_{0}}{4}, w_{2,1}=w_{1}+\frac{w_{2}-w_{0}}{4} .
\end{aligned}
$$

Show that in this case the conic $\operatorname{arcs} \operatorname{arcs} \boldsymbol{p}_{1}(u)$ and $\boldsymbol{p}_{2}(u)$ are also smoothly joined at the point $\boldsymbol{p}_{1}$. For this purpose represent the conic $\operatorname{arcs} \boldsymbol{p}_{1}(u)$ and $\boldsymbol{p}_{2}(u)$ as follows:

$$
\boldsymbol{p}_{i}(u)=\frac{\boldsymbol{r}_{i}(u)}{r_{i}(u)}, i \in\{1,2\}
$$

where

$$
\begin{aligned}
& \boldsymbol{r}_{1}(u)=(1-u)^{2} w_{0} \boldsymbol{p}_{0}+2(1-u) u w_{1,1} \boldsymbol{p}_{1,1}+u^{2} w_{1} \boldsymbol{p}_{1} \\
& \boldsymbol{r}_{2}(u)=(1-u)^{2} w_{1} \boldsymbol{p}_{1}+2(1-u) u w_{2,1} \boldsymbol{p}_{2,1}+u^{2} w_{2} \boldsymbol{p}_{2}
\end{aligned}
$$

and

$$
\begin{aligned}
& r_{1}(u)=(1-u)^{2} w_{0}+2(1-u) u w_{1,1}+u^{2} w_{1}, \\
& r_{2}(u)=(1-u)^{2} w_{1}+2(1-u) u w_{2,1}+u^{2} w_{2} .
\end{aligned}
$$

It follows from Equations (5.4) that the first two derivatives of the numerators $\boldsymbol{r}_{i}(u)$ and denominators $r_{i}(u)$ satisfy the following conditions at the common point $\boldsymbol{p}_{1}$ :

$$
\boldsymbol{r}_{1}^{\prime}(1)=\boldsymbol{r}_{2}^{\prime}(0), \boldsymbol{r}_{1}^{\prime \prime}(1)=\boldsymbol{r}_{2}^{\prime \prime}(0)
$$

and

$$
r_{1}^{\prime}(1)=r_{2}^{\prime}(0), r_{1}^{\prime \prime}(1)=r_{2}^{\prime \prime}(0)
$$

It is obvious that any of all other higher order derivatives of the numerators $\boldsymbol{r}_{i}(u)$ and denominators $r_{i}(u)$ is equal to zero. Now transform Equation (5.7) as follows: 


$$
r_{i}(u) \boldsymbol{p}_{i}(u)=\boldsymbol{r}_{i}(u), i \in\{1,2\}
$$

and find the first derivatives of the equation parts. It is obtained that

$$
r_{i}^{\prime}(u) \boldsymbol{p}_{i}(u)+r_{i}(u) \boldsymbol{p}_{i}^{\prime}(u)=\boldsymbol{r}_{i}^{\prime}(u), i \in\{1,2\}
$$

It follows from these equations taking into account Equations (5.8) and (5.9) that

$$
\boldsymbol{p}_{1}^{\prime}(1)=\frac{\boldsymbol{r}_{1}^{\prime}(1)-r_{1}^{\prime}(1) \boldsymbol{p}_{1}(1)}{r_{1}(1)}=\frac{\boldsymbol{r}_{2}^{\prime}(0)-r_{2}^{\prime}(0) \boldsymbol{p}_{2}(0)}{r_{2}(0)}=\boldsymbol{p}_{2}^{\prime}(0) .
$$

Analogously it can be proven that

$$
\boldsymbol{p}_{1}^{\prime \prime}(1)=\boldsymbol{p}_{2}^{\prime \prime}(0) \text {. }
$$

Now notice that all higher order derivatives of the conic $\operatorname{arcs} \boldsymbol{p}_{i}(u)$ at the common point $\boldsymbol{p}_{1}$ depends only values of these parametric curves at the common point and the first two derivatives of the numerators $\boldsymbol{r}_{i}(u)$ and denominators $r_{i}(u)$ at the common point. Then taking into account the last two equations it can be stated that Equations (5.3) are also fulfilled. Thus it is obtained that the conic arcs $\boldsymbol{p}_{1}(u)$ and $\boldsymbol{p}_{2}(u)$ are smoothly joined at the common point $\boldsymbol{p}_{1}$.

Since spline curves will be constructed by means of blending conic arcs it is reasonable to consider shape modification of the conic arc by means of changing weights of its knot points. This modification behaves just opposite to the modification of a weight of the conic control point. That is if a weight of the conic control point increases then the conic pulls toward the control point. Otherwise if a weight of the conic control point decreases then the conic pushes away from the control point. Figure 3 shows modification of a conic shape depending on a weight of its control point. Values of the weight are depicted near the knot points.

On the other hand if a weight of the conic knot point increases then the conic pushes away from the control point. Otherwise if a weight of the conic knot point decreases then the conic pulls toward the control point. Figure 4 shows modification of a conic shape depending on a weight of its knot point.

Now it is clear how weights of knot points influence on shape of two smoothly joined conics. Figure 5 shows modification of two smoothly joined conic shape by means of the weight which is prescribed to the knot point.

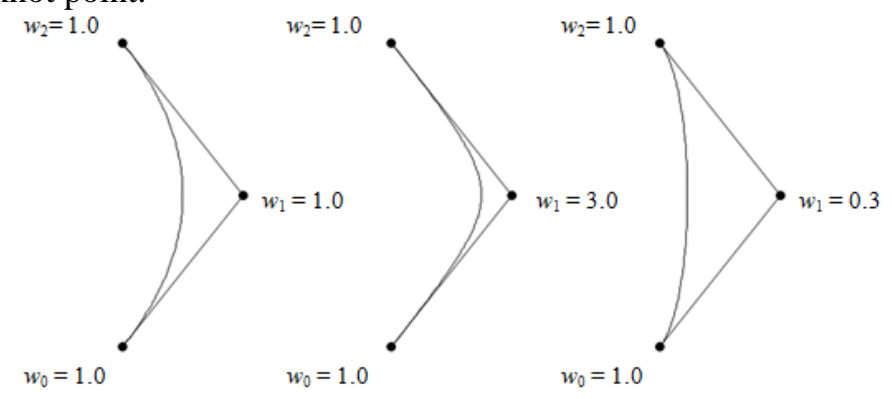

Figure 3. Modification of conic shape by means of a control point weight 
International Journal of Computer Graphics \& Animation (IJCGA) Vol.3, No.4, October 2013

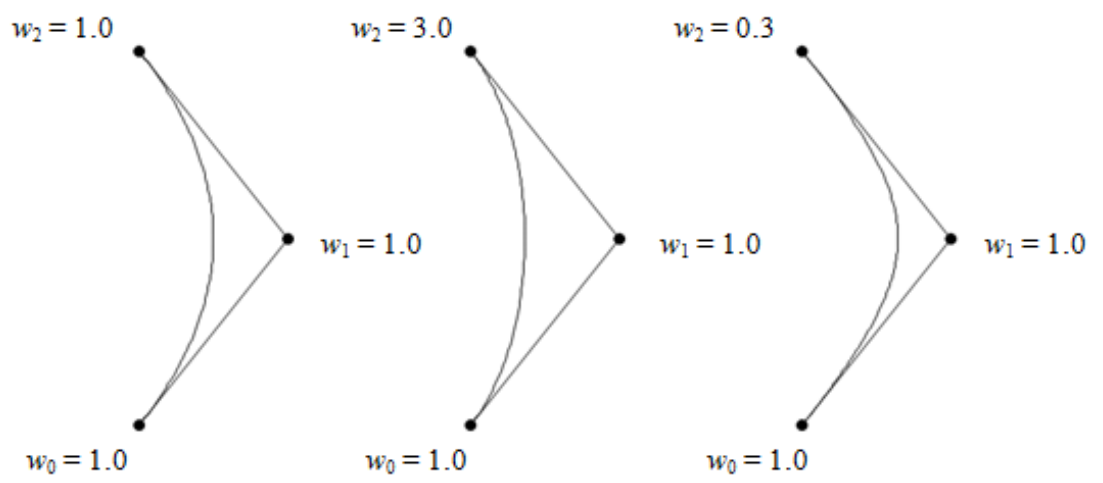

Figure 4. Modification of conic shape by means of a knot point weight

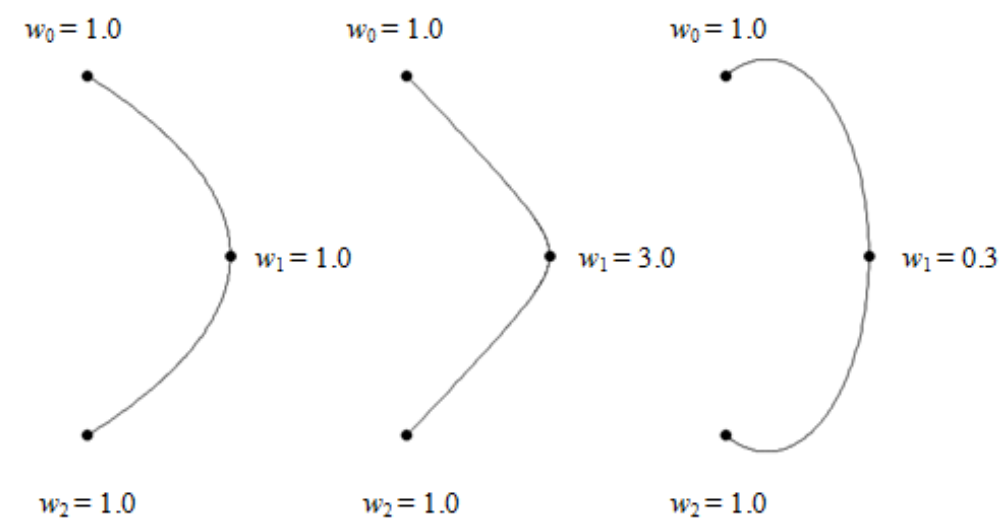

Figure 5. Modification of two smoothly joined conic shape by means of weights

More detailed considerations concerned with modification of rational curve shape by means of weights are presented in the works of Piegl [19], Sánchez-Reyes [20] and Juhász [21].

\section{Rational Bézier Spline Curves with local Shape Control}

The purpose of this section is to present a technique for construction of a rational spline curve $\boldsymbol{p}(u) \in C^{n}, n \in N$, which interpolates a sequence of knot points $\boldsymbol{p}_{i}, i \in\{0,1,2, \ldots, k\}, k \in N$, with the corresponding weights $w_{i}$. In order to solve the problem construct a segment $\boldsymbol{p}_{i}(u), 0<i<\mathrm{k}$, of the parametric curve $\boldsymbol{p}(u)$ by means of blending two conic $\operatorname{arcs} \boldsymbol{p}_{i, 1}(u)$ and $\boldsymbol{p}_{i, 2}(u)$ as was proposed in Section 3. Figure 6 explains construction a segment $\boldsymbol{p}_{i}(u)$ of the spline curve $\boldsymbol{p}(u)$.

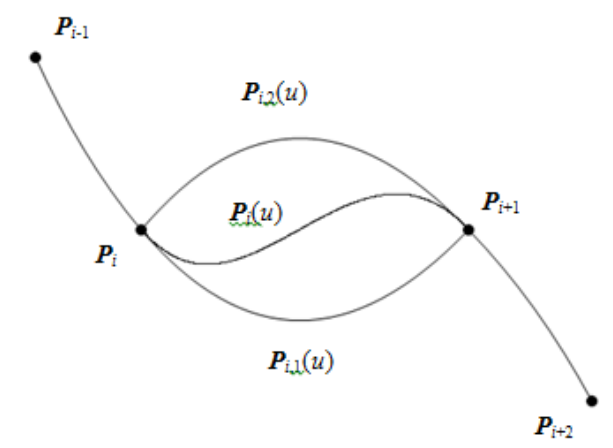

Figure 6. Construction of a spline curve segment 
In order to ensure $C^{n}$ smoothness of the spline curve $\boldsymbol{p}(u)$ it is necessary to ensure that that the conic arcs $\boldsymbol{p}_{i-1,1}(u)$ and $\boldsymbol{p}_{i, 2}(u)$ are smoothly joined at the common point $\boldsymbol{p}_{i}$. This can be obtained by constructing the conic arcs $\boldsymbol{p}_{i-1,1}(u)$ and $\boldsymbol{p}_{i, 2}(u)$ as was proposed in Section 5. Taking into account these considerations define the conic $\operatorname{arcs} \boldsymbol{p}_{i, 1}(u)$ and $\boldsymbol{p}_{i, 2}(u)$ as follows:

$$
\boldsymbol{p}_{i, 1}(u)=\frac{(1-u)^{2} w_{i} \boldsymbol{p}_{i}+2(1-u) u w_{i, 1} \boldsymbol{p}_{i, 1}+u^{2} w_{i+1} \boldsymbol{p}_{i+1}}{(1-u)^{2} w_{i}+2(1-u) u w_{i, 1}+u^{2} w_{i+1}}, u \in[0,1]
$$

for $i \in\{0,1, \ldots, k-1\}$ and

$$
\boldsymbol{p}_{i, 2}(u)=\frac{(1-u)^{2} w_{i} \boldsymbol{p}_{i}+2(1-u) u w_{i, 2} \boldsymbol{p}_{i, 2}+u^{2} w_{i+1} \boldsymbol{p}_{i+1}}{(1-u)^{2} w_{i}+2(1-u) u w_{i, 2}+u^{2} w_{i+1}}, u \in[0,1] .
$$

for $i \in\{1,2, \ldots, k\}$ where the control points $\boldsymbol{p}_{i, 1}$ and $\boldsymbol{p}_{i, 2}$ with the corresponding weights $w_{i, 1}$ and $w_{i, 2}$ are defined using Equations

$$
\begin{aligned}
& \boldsymbol{p}_{i, 1}=w_{i} \boldsymbol{p}_{i}-\frac{w_{i+1} \boldsymbol{p}_{i+1}-w_{i-1} \boldsymbol{p}_{i-1}}{4}, \boldsymbol{p}_{i, 2}=w_{i} \boldsymbol{p}_{i}+\frac{w_{i+1} \boldsymbol{p}_{i+1}-w_{i-1} \boldsymbol{p}_{i-1}}{4} . \\
& w_{i, 1}=w_{i}-\frac{w_{i+1}-w_{i-1}}{4}, w_{i, 2}=w_{i}+\frac{w_{i+1}-w_{i-1}}{4} .
\end{aligned}
$$

Then a segment $\boldsymbol{p}_{i}(u)$ of the spline curve $\boldsymbol{p}(u)$ can be defined using Equation (3.5) as follows:

$$
\boldsymbol{p}_{i}(u)=\frac{\left(1-w_{n}(u)\right) \boldsymbol{r}_{i, 1}(u)+w_{n}(u) \boldsymbol{r}_{i, 2}(u)}{\left(1-w_{n}(u)\right) r_{i, 1}(u)+w_{n}(u) r_{i, 2}(u)}, u \in[0,1],
$$

where

$$
\begin{aligned}
& \boldsymbol{r}_{i, j}(u)=(1-u)^{2} w_{i} \boldsymbol{p}_{i}+2(1-u) u w_{i, j} \boldsymbol{p}_{i, j}+u^{2} w_{i+1} \boldsymbol{p}_{i+1}, j \in\{1,2\} \\
& r_{i, j}(u)=(1-u)^{2} w_{i}+2(1-u) u w_{i, j}+u^{2} w_{i+1}, j \in\{1,2\}
\end{aligned}
$$

It follows from Equations (3.3) and (5.3) that in this case segments of the parametric curve $\boldsymbol{p}(u)$ satisfy the following condition:

$$
\boldsymbol{p}_{i}^{(n)}(1)=\boldsymbol{p}_{i+1}{ }^{(n)}(0), n \in N
$$

Figure 7 shows how a shape of the spline curve depends on the weight prescribed to the knot point of the spline curve. Weights of all other knot points of the spline curve are equal to the unity.

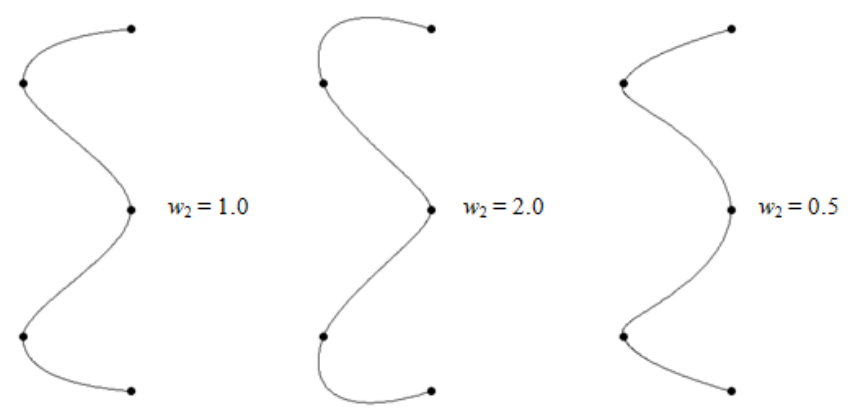

Figure 7. Modification of a spline curve shape by means of weights 
International Journal of Computer Graphics \& Animation (IJCGA) Vol.3, No.4, October 2013

\section{Conclusions}

The approach to construction of $C^{n}$ continuous interpolating spline curves by means of blending rational quadric Bézier curves is introduced. Spline curves are constructed locally which implies local shape control of them by means of weights which are assigned to the knot points of the constructed spline curve. Bézier representation of the considered spline curves is introduced. The presented technique can be used for fast prototyping rational spline Bézier. Besides since the spline curves are constructed locally the presented technique can be also used in real-time geometric applications connected with computer graphics and animation.

\section{REFERENCES}

[1] Pobegailo, A. P., Polynomial blending of parametric curves in linear spaces. BSU, Minsk, 2013.

[2] Overhauser, A.W., (1968) “Analytic Definition of Curves and Surfaces by Parabolic Blending”, Tech. Rep. No. SL68-40, Ford Motor Company Scientific Laboratory.

[3] Rogers, D.F., Adams, J.A., (1989) Mathematical elements for computer graphics, McGraw-Hill publisher.

[4] Chuan Sun, Huanxi Zhao, (2009) "Generating fair, C2 continuous splines by blending conics", Computers \& Graphics, Vol. 33, No. 2, pp. 173-180.

[5] Hartmann, E., (2001) "Parametric $G^{n}$ blending of curves and surfaces", The Visual Computer, Vol. 17, No. 1, pp. 1-13.

[6] Meek, D.S., Walton, D. J., (2009) "Blending two parametric curves", Computer-Aided Design, Vol. 41, No. 6, pp. 423-431.

[7] Tai, C.-L., Barsky, B.A. and Loe, K.F., (2000) "An interpolation method with weights and relaxation parameters", in A. Cohen et al. (eds.), Curves and surface ftting, Saint-Malo, Vanderbilt Univ. Press, Nashville, pp. 393-402.

[8] Habib, Z., Sakai, M. and Sarfraz, M., (2004) "Interactive Shape Control with Rational Cubic Splines", Computer-Aided Design \& Applications, Vol. 1, No. 1-4, pp. 709-718.

[9] Habib, Z., Sarfraz, M. and Sakai, M., (2005) "Rational cubic spline interpolation with shape control", Computers \& Graphics, Vol. 29, No. 4, pp. 594-605.

[10] Sarfraz, M., (1994) "Generalized geometric interpolation for rational cubic splines", Computers \& Graphics, Vol. 18, No. 1, pp. 61-72.

[11] Karčiauskas, K., Peters, J., (2011) “Rational G2 splines”, Graphical Models, Vol. 73, No. 5, pp. 286295.

[12] Fangxun Bao, Qinghua Sun, Qi Duan, (2009) "Point control of the interpolating curve with a rational cubic spline", Journal of Visual Communication and Image Representation, Vol. 20, No. 4, pp. 275280 .

[13] Qi Duan, Djidjeli, K., Price, W.G., Twizell, E.H., (2000) "Weighted rational cubic spline interpolation and its application", Journal of Computational and Applied Mathematics, Vol. 117, No. 2, pp. 121-135.

[14] Qi Duan, Fangxun Bao, Shitian Du, Twizell, E.H., (2009) "Local control of interpolating rational cubic spline curves”, Computer-Aided Design, Vol. 41, No. 11, pp. 825-829.

[15] Qi Duana, Liqiu Wangb, Twizellc, E.H., (2005) “A new weighted rational cubic interpolation and its approximation", Applied Mathematics and Computation, Vol. 168, No. 2, pp. 990-1003.

[16] Pobegailo, A.P., (1991) "Local interpolation with weight functions for variable-smoothness curve design”, Computer-Aided Design, Vol. 23, No. 8, pp. 579-582.

[17] Pobegailo, A.P., (1992) "Geometric modeling of curves using weighted linear and circular segments", The Visual Computer, Vol. 8, No. 4, pp. 241-245. 
[18] Wiltsche, A., (2005) "Blending curves", Journal for Geometry and Graphics, Vol. 9, No. 1, pp. 6775.

[19] Piegl, L., (1989) "Modifying the shape of rational B-splines. Part 1: curves", Compuer.-Aided Design, Vol. 21, No. 8, pp. 509-518.

[20] Sánchez-Reyes, J., (1997) “A Simple Technique for NURBS Shape Modification”, IEEE Computer Graphics and Applications, Vol. 17, No. 1, pp. 52-59.

[21] Juhász, I., (1999) "Weight-based shape modification of NURBS curves", Computer Aided Geometric Design, Vol. 16, No. 5, pp. 377-383.

\section{Authors}

A. P. Pobegailo graduated from Belarusian State University in 1981. Then he obtained Ph. Degree on system analysis and automatic control. Currently he is an assistant professor at Belarusian State University. He teaches courses on operating systems and systems design. His research interests include computer graphics and geometric design.

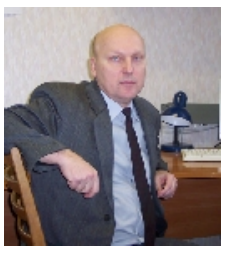

